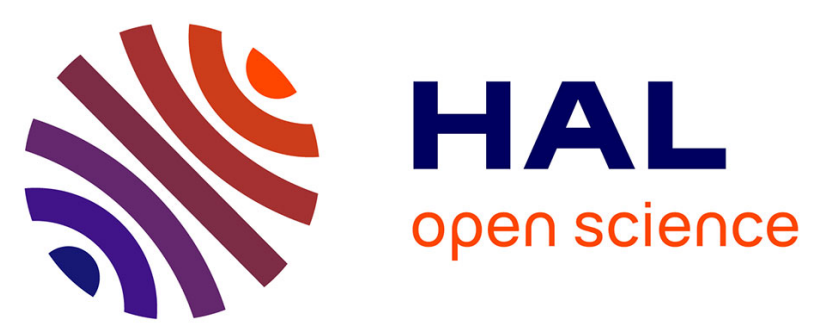

\title{
Biologie des pucerons des céréales dans l'Ouest de la France. VI. - Etude comparative des fluctuations au champ des populations de Sitobion avenae (F.), Metopolophium dirhodum (Wlk.) et Rhopalosiphum padi (L.) sur différents cultivars de blé d'hiver
}

Charles-Antoine Dedryver, Jean-Paul Di Pietro, Augustine Gellé, Sylvie Tanguy

\section{To cite this version:}

Charles-Antoine Dedryver, Jean-Paul Di Pietro, Augustine Gellé, Sylvie Tanguy. Biologie des pucerons des céréales dans l'Ouest de la France. VI. - Etude comparative des fluctuations au champ des populations de Sitobion avenae (F.), Metopolophium dirhodum (Wlk.) et Rhopalosiphum padi (L.) sur différents cultivars de blé d'hiver. Agronomie, 1986, 6 (1), pp.75-84. hal-00884850

\author{
HAL Id: hal-00884850 \\ https://hal.science/hal-00884850
}

Submitted on 1 Jan 1986

HAL is a multi-disciplinary open access archive for the deposit and dissemination of scientific research documents, whether they are published or not. The documents may come from teaching and research institutions in France or abroad, or from public or private research centers.
L'archive ouverte pluridisciplinaire HAL, est destinée au dépôt et à la diffusion de documents scientifiques de niveau recherche, publiés ou non, émanant des établissements d'enseignement et de recherche français ou étrangers, des laboratoires publics ou privés. 


\title{
Biologie des pucerons des céréales dans l'Ouest de la France. VI. - Etude comparative des fluctua- tions au champ des populations de Sitobion ave- nae (F.), Metopolophium dirhodum (Wlk.) et Rhopalosiphum padi (L.) sur différents culti- vars de blé d'hiver
}

\author{
Charles-Antoine DEDRYVER \& Jean-Paul DI PIETRO \\ avec la collaboration technique d'Augustine Gellé \& Sylvie TANGUY \\ I.N.R.A., Laboratoire de Recherches de la Chaire de Zoologie de l'E.N.S.A. de Rennes, F 35650 Le Rheu
}

Au cours d'expérimentations effectuées en champs de 1978 à 1982 dans le Bassin de Rennes (Ouest de la France), nous avons comparé l'évolution des populations de $S$. avenae, $M$. dirhodum et $R$. padi sur différentes variétés de blé d'hiver. Les différences intervariétales sont essentiellement significatives dans les cas de $S$. avenae et de $R$. padi, et ceci, après l'épiaison. Chez $S$. avenae seulement, des différences intervariétales significatives se manifestent au moment des maximums de population. Parmi les variétés testées « Maris Huntsman » est significativement plus infestée que les autres par au moins 2 sinon 3 espèces de pucerons. Par contre, « Roazon " est le plus souvent moins infestée que la plupart des autres variétés. Pour beaucoup d'autres cultivars, les différences observées sont instables selon les années, y compris pour « Mironowskaïa 808 ». La variété « Fidel », qui n'a été testée que 2 années, paraît peu favorable à $S$. avenae.

La comparaison des courbes d'évolution des populations de $S$. avenae sur " Maris Huntsman » et " Roazon " de 1978 à 1982 permet d'avancer l'hypothèse de la conjonction de 3 facteurs pouvant expliquer les différences observées : une résistance partielle vraie de type antibiosis, un effet de la précocité de la variété, un rôle de l'état pathologique du cultivar. Les effets variétaux observés au champ ne sont cependant pas du même ordre de grandeur que ceux produits, en régions océaniques, par des modifications de la date de semis d'un même cultivar.

Mots clés additionnels : Aphides, Triticum aestivum, effet variétal, résistance. populations of Sitobion avenae (F.), Metopolophium dirhodum (Wlk.) and Rhopalosiphum padi (L.), on different winter wheat cultivars.

Experiments set up in fields from 1978 to 1982 in the Rennes Basin (western part of France) allowed the comparison of the development of $S$. avenae, $M$. dirhodum and $R$. padi on different cultivars of winter wheat. The differences between varieties were mainly significant for $S$. avenae and $R$. padi and appeared after heading. Differences were significant at the population maximum only in the case of $S$. avenae. Among the tested cultivars, "Maris Huntsman " was regularly more infested than the others by at least 2 (S. avenae and $R$. padi) and sometimes all 3 species of aphids. In contrast "Roazon " was generally less infested than most other varieties. For many other cultivars, the differences observed were not stable from year to year, including « Mironowskaya 808 ». « Fidel » seemed to be less favourable to $S$. avenae but was only tested during 2 years. Comparison of the development curves of $S$. avenae on "Maris Huntsman " and "Roazon " over 5 years suggested that the origins of the observed differences could be partial antibiosis, cultivar precocity and pathological state. Varietal effects on aphids, observed in the field, did not seem, in the west of France, to be as large as the effects of different sowing dates of the same cultivar.

Additional key words : Aphids, Triticum aestivum, varietal effect, resistance. 


\section{INTRODUCTION}

Les populations de pucerons des céréales fluctuent sous la dépendance, entre autres, de facteurs biotiques tels que ceux relatifs aux plantes-hôtes (blé, orge, avoine, maïs, graminées fourragères ou adventices) et à leurs conditions de culture (fertilisation, époque de semis). A l'occasion d'observations de terrain, de nombreux auteurs se sont posé la question de l'existence d'effets variétaux dans le cas des céréales à paille et du maïs. Ces effets variétaux pourraient se traduire par une plus ou moins grande tolérance de différents cultivars aux attaques de pucerons (HINZ \& DAEBELLER, 1976) ou/et par un caractère plus ou moins favorable de ces cultivars vis-à-vis des pucerons (LATTEUR, 1976 ; DUfOUR, 1982). Ces études sont motivées par l'intérêt qu'il y aurait à établir un classement des variétés existantes entre les plus favorables et les plus sensibles aux pucerons et celles qui le sont le moins, puis à en sélectionner de nouvelles présentant des niveaux de "résistance " accrus. De plus, une meilleure connaissance de l'effet variétal sur la multiplication des pucerons permettrait d'apporter un élément d'explication supplémentaire aux fréquentes pullulations observées en Europe depuis une quinzaine d'années en confirmant ou infirmant l'hypothèse émise par certains auteurs selon laquelle les cultivars anciens étaient moins favorables aux pucerons que les variétés nouvelles qui les ont remplacés (SOTHERTON \& VAN EMDEN, 1982).

Le problème de la "tolérance ", qui se pose en terme de rendements obtenus face à une infestation standard de pucerons, a été relativement peu étudié du fait de sa complexité. Il n'en est pas de même de celui qui consiste à évaluer la plus ou moins grande aptitude de différents cultivars à multiplier ces pucerons.

Jusqu'à présent, les travaux les plus nombreux ont été faits aux Etats-Unis et ont porté sur la résistance à différents biotypes de Schizaphis graminum Rond., tant en ce qui concerne le blé (HARVEY et al., 1980; JOPPA et al., 1980) que le maïs et surtout le sorgho (MORGAN et al., 1980 ; CAMPBELl et al., 1982).

En Europe, c'est la résistance des céréales à paille et du maïs à Sitobion avenae F., Metopolophium dirhodum Wlk. et Rhopalosiphum padi L. qui a fait l'objet de recherches, entreprises le plus souvent selon 2 démarches différentes et complémentaires :

- la première consiste à isoler d'éventuels effets variétaux par des expérimentations en conditions contrôlées ou semi-contrôlées, généralement en serre (HINZ \& DAEBELLER, 1974; LOWE, 1978, 1980, $1981 a$ et $b, 1982$; SOTHERTON \& VAN EMDEN, 1982);

- la seconde, beaucoup plus rarement employée, permet de mesurer la résultante au champ de ces effets variétaux et des autres facteurs influant sur la dynamique des populations de pucerons des céréales, de manière à cerner l'importance pratique des premiers (LATTEUR, 1976 ; DUfOUR, 1982 ; CHAUVEAU, comm. pers.).

Nous présentons ici le résultat d'expérimentations appartenant à cette $2^{\mathrm{e}}$ catégorie, effectuées au champ, dans le Bassin de Rennes, au cours desquelles nous avons voulu estimer, pour quelques cultivars de blé d'hiven choisis parmi les plus cultivés en France en 1978, d'une part, l'importance de l'effet variétal sur les pucerons et, d'autre part, la stabilité au cours des ans des différences observées.

\section{MATÉRIEL ET MÉTHODES}

Les expérimentations ont été faites d'octobre 1977 à juin 1982 sur le domaine du Centre de Recherches de l'I.N.R.A. au Rheu (Ille-et-Vilaine). Les cultivars testés et les plans d'expérience sont indiqués au tableau 1. En 1979, 1980 et 1982, il y a eu 2 essais distincts semés à des dates différentes, début octobre et début novembre. En 1980, le plan d'expérience de la $1^{\text {re }}$ date de semis ne comportait que 2 répétitions en bandes alternées et n'a donc pu être pris en compte lors des tests statistiques.

L'estimation des populations de pucerons est effectuée à partir de prélèvements de talles faits chaque semaine dans les parcelles et ramenés au laboratoire pour dépouillement. Selon les types de plans d'expérience et le degré d'infestation des blés par les pucerons, un nombre variable de talles consécutives est prélevé dans 5 placettes régulièrement réparties dans chaque parcelle, le total récolté étant toujours compris entre 100 et 800 talles par cultivar et par date de prélèvement. Les pucerons sont conservés dans de l'alcool à $70^{\circ}$ et déterminés à la loupe binoculaire.

Certaines des variétés de blé testées («Capitole», " Champlein », « Maris Huntsman », « Roazon » et "Joss ») ont été choisies parmi celles qui étaient les plus cultivées en France en 1977-1978 et de manière à couvrir une gamme de précocités différentes. D'autres ont été retenues en fonction de leurs caractères botaniques particuliers : épi très fortement aristé dans le cas du cultivar "Fidel », apparence vert-bleuté de la culture dans le cas des lignées $\mathrm{R} 51$ et R 37. D'autres, comme "Mironowskaïa 808 ", sont des cultivars déjà signalés en Europe de l'Est comme peu favorables aux pucerons (HINZ \& DAEBELLER, 1974 ; 1976). Enfin, en 1981 et 1982, d'autres variétés ("Caucase ») ou lignées issues de croisements effectués par le laboratoire du blé de la Station d'Amélioration des Plantes de l'I.N.R.A. du Rheu ont été testées, au champ et au laboratoire (DI PIETRO \& DEDRYVER, 1984). Ces lignées sont apparentées, soit à " Mironowskaïa 808 " (qui est utilisé comme pourvoyeur de gènes de résistance au froid et à la septoriose), soit à d'autres cultivars ou géniteurs intéressants en sélection, tel le blé «Loros », résistant au nématode à kystes des céréales Heterodera avenae Wlb. Les stades du blé sont repérés selon l'échelle de Zadoks (ZADOKS et al., 1977).

\section{RÉSULTATS}

\section{A. Evolution au cours du temps des différences inter- variétales}

Pour chaque plan d'expérience, dont le nombre de blocs était au moins égal à 3 , une analyse de la variance à 2 facteurs a été effectuée sur les données transformées en $\log (n+1)$, $n$ étant le nombre de pucerons d'une espèce, par échantillon de 20 talles, pour un prélèvement, sur un cultivar donné. Les analyses ont ainsi été faites sur l'expérimentation de 1978 (semis précoce), sur les 2 expérimentations de 
TABLEAU 1

Caractéristiques des plans d'expériences mis en place de 1978 à 1982.

Characteristics of the experiments set up in 1978-1982.

\begin{tabular}{|c|c|c|c|c|c|}
\hline & 1978 & 1979 & 1980 & 1981 & 1982 \\
\hline \multirow[t]{9}{*}{ Cultivars testés } & $\begin{array}{l}\text { Capitole } \\
\text { Champlein }\end{array}$ & Champlein & $\begin{array}{l}\text { Capitole } \\
\text { Champlein }\end{array}$ & $\begin{array}{l}\text { Capitole } \\
\text { Champlein } \\
\text { Fidel }\end{array}$ & $\begin{array}{l}\text { Capitole } \\
\text { Champlein } \\
\text { Fidel }\end{array}$ \\
\hline & Maris Huntsman & Maris Huntsman & Maris Huntsman & Maris Huntsman & Maris Huntsman \\
\hline & Roazon & Roazon & Roazon & Roazon & Roazon \\
\hline & & Mironowskaïa 808 & Mironowskaïa 808 & $\begin{array}{l}\text { Mironowskaïa } 808 \\
\text { Caucase }\end{array}$ & $\begin{array}{l}\text { Mironowskaïa } 808 \\
\text { Caucase }\end{array}$ \\
\hline & Joss & & & L 707 & L 707 \\
\hline & & & & $\begin{array}{l}\text { L } 755 \\
\text { R } 51\end{array}$ & L 755 \\
\hline & & & & & $\mathrm{Mi} \times \mathrm{B} .104 .2 .1$ \\
\hline & & & & & $\begin{array}{l}\text { Mi } \times \text { B.5.4.3 } \\
\text { A } 82.2 \\
\text { A } 207.4 \\
\text { Loros } \times \text { Cap. } \times \text { V M } 8\end{array}$ \\
\hline & 5 & 4 & 5 & 10 & 15 \\
\hline $\begin{array}{l}\text { Semis précoce } \\
\text { (début octobre) }\end{array}$ & 1 & 1 & 1 & 1 & 1 \\
\hline $\begin{array}{l}\text { Semis tardif } \\
\text { (début novembre) }\end{array}$ & - & 1 & 1 & - & 1 \\
\hline Plan d'expérience & $\begin{array}{c}\text { Bloc de Fischer } \\
3 \text { blocs }\end{array}$ & $\begin{array}{l}\text { Bloc de Fischer } \\
4 \text { blocs }\end{array}$ & $\begin{array}{l}\text { Bandes alternées } \\
4 \text { blocs * }\end{array}$ & $\begin{array}{l}\text { Bloc de Fischer } \\
4 \text { blocs }\end{array}$ & $\begin{array}{l}\text { Bloc de Fischer } \\
4 \text { blocs }\end{array}$ \\
\hline Taille des parcelles & $50 \mathrm{~m}^{2}$ & $20 \mathrm{~m}^{2}$ & $150 \mathrm{~m}^{2}$ & $7 \mathrm{~m}^{2}$ & $7 \mathrm{~m}^{2}$ \\
\hline $\begin{array}{l}\text { Nombre minimum et } \\
\text { maximum de talles pré- } \\
\text { levées à chaque date par } \\
\text { variété }\end{array}$ & 300 & $200-800$ & $200-800$ & $100-400$ & $100-400$ \\
\hline
\end{tabular}

1979 (semis précoce et semis tardif), sur le semis tardif de 1980, sur l'expérimentation de 1981 (semis précoce) et sur les 2 expérimentations de 1982 (semis précoce et semis tardif). Pour les 2 expérimentations de 1979 et pour le semis tardif de 1982, aucune différence significative n'a été trouvée entre cultivars, quelle que soit l'espèce de puceron; ces cas ne sont donc pas indiqués sur les figures 1,2 et 3 qui représentent l'évolution au cours du temps du $\mathrm{F}$ de Fischer du facteur « variété » pour $S$. avenae, $R$. padi et $M$. dirhodum pour 4 expérimentations effectuées en 1978, 1980, 1981 et 1982 :

+ Il n'y a pas de différences significatives entre variétés, quelle que soit l'espèce de puceron, au moment de la contamination des parcelles par les ailés, qui s'effectue soit à l'automne précédent (1978, 1981 et 1982), soit au printemps (1980, 1978 pour M. dirhodum).

+ Plus généralement, on ne trouve pas, ou très peu, de différences significatives entre cultivars avant l'épiaison, quelle que soit l'espèce de puceron. Les 2 exceptions concernent $R$. padi et surtout $S$. avenae en 1981.

+ A l'époque où les populations de pucerons sont maximales, il existe, dans tous les cas, des différences intervariétales significatives concernant les infestations par S. avenae, alors que ceci ne se produit qu'en 1980 pour $M$. dirhodum et en 1978 pour $R$. padi.
+ Pour les 3 espèces de pucerons, on trouve des différences intervariétales significatives lorsque les populations sont en phase décroissante. Ces différences, pour une même année, sont plus marquées dans les cas de $S$. avenae et $R$. padi que dans celui de M. dirhodum.

L'analyse indique que l'effet "variétal » des cultivars de blé d'hiver sur les pucerons est décelable au champ 4 années sur 5 , mais qu'il se manifeste assez tardivement et est particulièrement marqué sur les populations de pucerons qui atteignent leur maximum de développement nettement après l'épiaison, c'est-àdire appartenant à l'espèce $S$. avenae.

\section{B. Comparaison de l'infestation des différents culti- vars}

Des tests de comparaisons multiples de moyennes de DUNCAN ont été effectués sur les moyennes marginales du facteur "variété " pour les populations des 3 espèces de pucerons, lorsqu'une telle analyse était possible, c'est-à-dire lorsque le $F$ de Fischer était significatif. En 1982, seules 10 variétés sur 15 (les mêmes qu'en 1981) ont été prises en considération. Les résultats sont indiqués sur les figures 4,5 et 6 . Le nombre de symboles dans chaque case des tableaux à double entrée indique le nombre de prélèvements au 


\section{S. avenae}

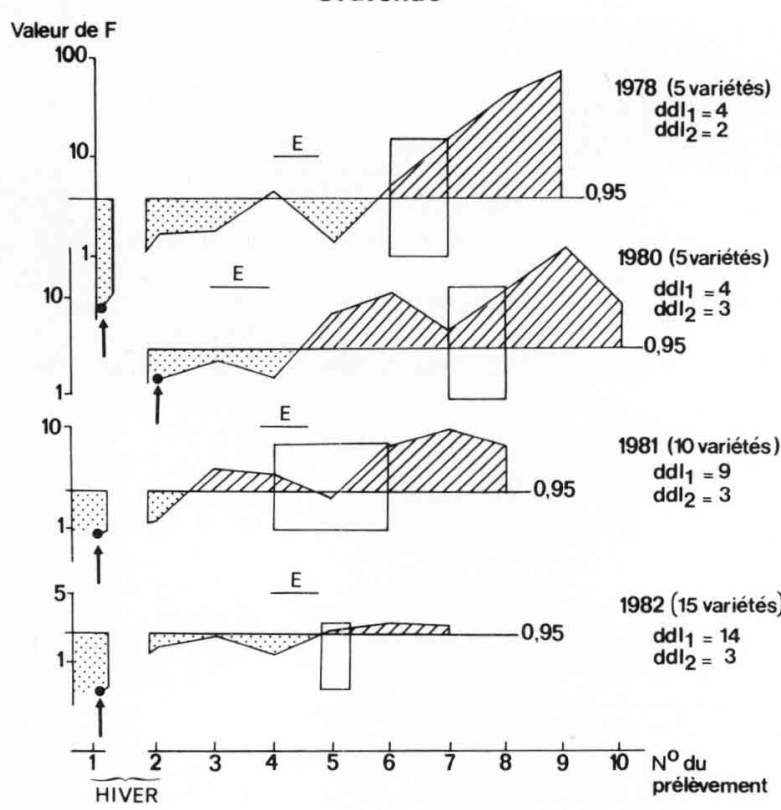

Figure 1

Evolution au cours du temps du F de Fisher du facteur "variété » pour S. avenae pour 4 expérimentations effectuées en 1978, 1980, 1981 et 1982.

Z7य $F$ de Fisher significatif au seuil $5 \%$.

$F$ de Fisher non significatif au seuil $5 \%$.

$\rightarrow$ Période approximative de contamination des parcelles.

$\underline{E}$ Epiaison.

Période où les populations sont maximales.

ddll Nombre de degrés de liberté du facteur "variété ".

dd12 Nombre de degrés de liberté du facteur " bloc ».

Values of Fisher's $F$ for the factor « variety » for different dates of sampling, in the case of $\mathrm{S}$. avenae, for 4 experiments designed in 1978, 1980, 1981 and 1982.

Eat Significant $F$ values at $5 \%$.

Non significant $F$ values at $5 \%$

$\rightarrow$ Approximate period of infestation of the plots.

E Heading.

$\square$ Period of maximal populations.

ddl1 Degrees of freedom of the factor " variety ".

dd12 Degrees of freedom of the factor « block ».

cours desquels une différence significative entre 2 cultivars a été trouvée, le cultivar en colonne étant le plus contaminé, celui en ligne, le moins. La lecture des colonnes permet ainsi de visualiser les variétés significativement "très contaminées", à l'inverse, celle des lignes discrimine les variétés "peu contaminées ».

+ En 1978, les cultivars remarquables par leurs fortes populations de pucerons sont "Maris Huntsman » et, plus secondairement, "Joss ». Les cultivars peu infestés sont "Roazon " et "Capitole ». L'ensemble de ces remarques est valable pour les 3 espèces de pucerons.

+ En 1980 , les cultivars qui apparaissent globalement plus fortement infestés que les autres sont "Capitole », pour les 3 espèces de pucerons mais tout particulièrement par $S$. avenae, puis, plus secondairement, " Maris Huntsman » par S. avenae et M. dirhodum. A l'inverse, les cultivars peu infestés sont "Mironowskaïa 808 " dans le cas des 3 espèces de pucerons, et " Roazon » pour $S$. avenae et $M$. dirhodum.

+ En 1981, les cultivars très infestés par rapport aux autres sont "Maris Huntsman » pour les 3 espè-

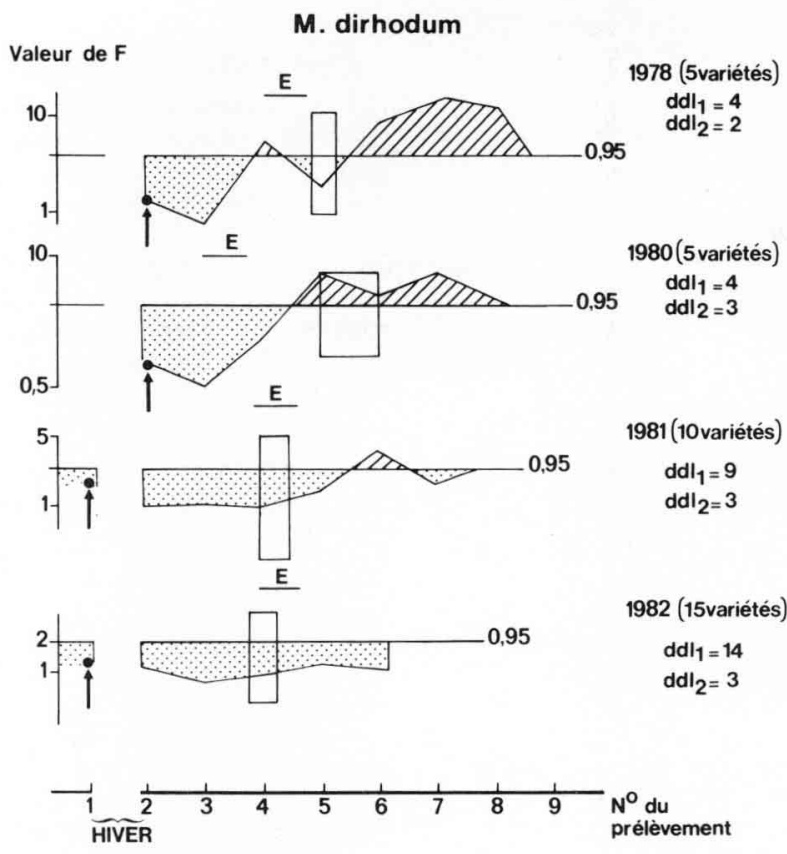

Figure 2

Même légende que pour la figure l pour M. dirhodum. As for figure 1 for $\mathrm{M}$. dirhodum.

R. padi

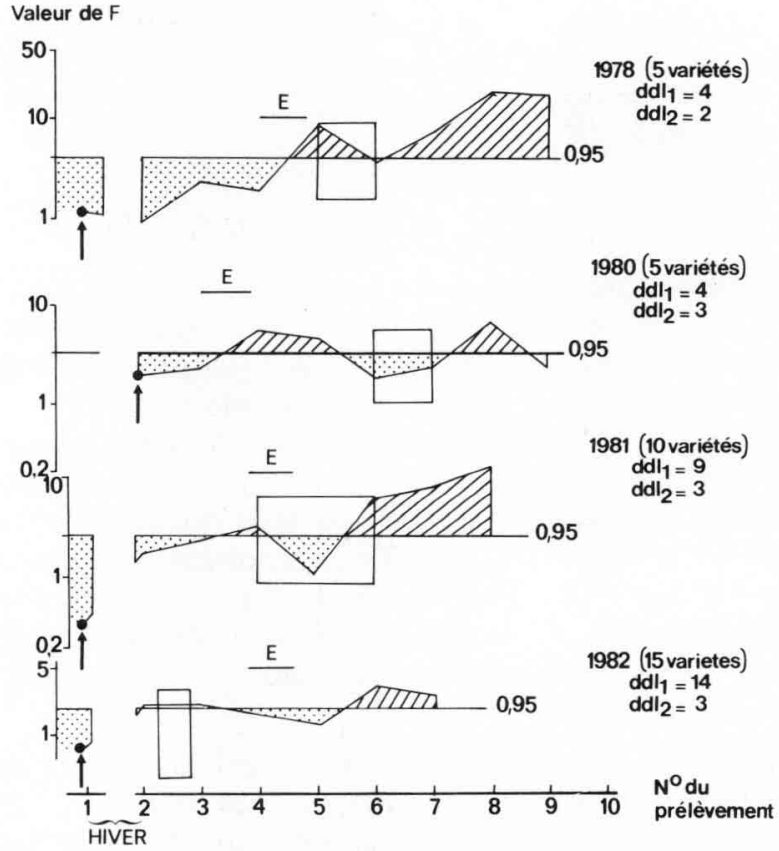

Figure 3

Même légende que pour la figure 1 pour $\mathrm{R}$. padi. As for figure 1 for R. padi.

ces de pucerons, "Capitole" pour $S$. avenae et $R$. padi, ainsi que $\mathrm{R} 51$ pour $S$. avenae. Les cultivars peu infestés sont "Fidel » et "Roazon » dans le cas de $S$. avenae, "Mironowskaïa 808 " et L 755 dans celui de $M$. dirhodum. Pour $R$. padi, il ne se dégage rien de clair dans le sens des faibles infestations. 


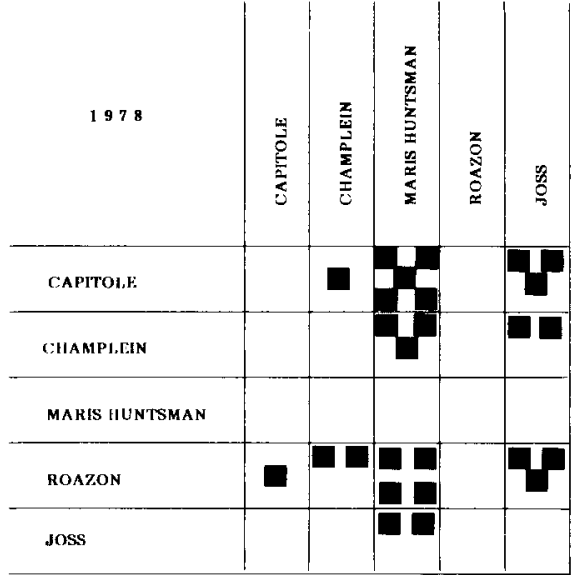

\begin{tabular}{|c|c|c|c|c|c|c|c|c|c|}
\hline 1981 & 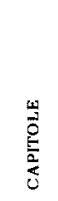 & 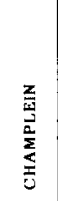 & 㞾 & 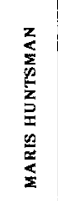 & 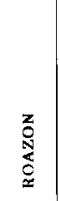 & $\stackrel{\overrightarrow{\dot{\theta}}}{\propto}$ & 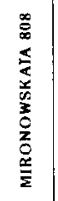 & 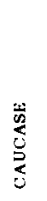 & 产 \\
\hline CAPITOLE & & & & $\mathbf{\square}$ & & $\square$ & & & \\
\hline CHAMPLEIN & & & & & a & ש & 口 & & \\
\hline FIDEL. & & & & & & & & & \\
\hline MARIS HUNTSMAN & 口 & $\mathbf{\square}$ & & & & & & & \\
\hline ROAZON & $\mathbf{\square \square}$ & $\overline{\square \square}$ & & & & & & & \\
\hline R 5.1 & $\square$ & a & & & a & & & & \\
\hline MIRONOWSKAтA 808 & $\mathbf{E}$ & 口 & & n & $\mathbf{\square}$ & & & $\mathbf{D}$ & \\
\hline CAUCASE & DE & & & D日 & & $\square$ & $\square$ & & \\
\hline L 707 & ש & ש & & 曰 & & $\square$ & & 10 & \\
\hline ᄂ 755 & $\mathbf{\square}$ & घ & & & & & $\boldsymbol{\theta}$ & a & \\
\hline
\end{tabular}

Figure 4

Tests de comparaison multiple de Duncan, sur les moyennes marginales du facteur " variété » pour $\mathrm{S}$. avenae. Le nombre de symboles dans chaque case des tableaux à double entrée indique le nombre de prélèvements au cours desquels une différence significative entre 2 cultivars a été trouvée. Le cultivar en colonne est le plus contaminé, celui en ligne, le moins.

+ En 1982, le cultivar « Maris Huntsman » est plus infesté que les autres par $S$. avenae et $R$. padi. Les cultivars peu infestés sont "Fidel ", dans le cas de $S$. avenae, et « Mironowskaïa 808 », dans celui de $R$. padi. Les comparaisons de moyennes n'ont pas été faites pour les populations de $M$. dirhodum car l'analyse de la variance ne permettait pas de dégager de différences intervariétales au sujet de cette espèce.

D'une manière générale, c'est pour les populations de $S$. avenae et de $R$. padi qu'on trouve le plus grand nombre de différences significatives entre cultivars, ce qui est logique puisque l'analyse de la variance avait précédemment montré que c'est pour ces 2 espèces que les différences intervariétales étaient le plus sou-
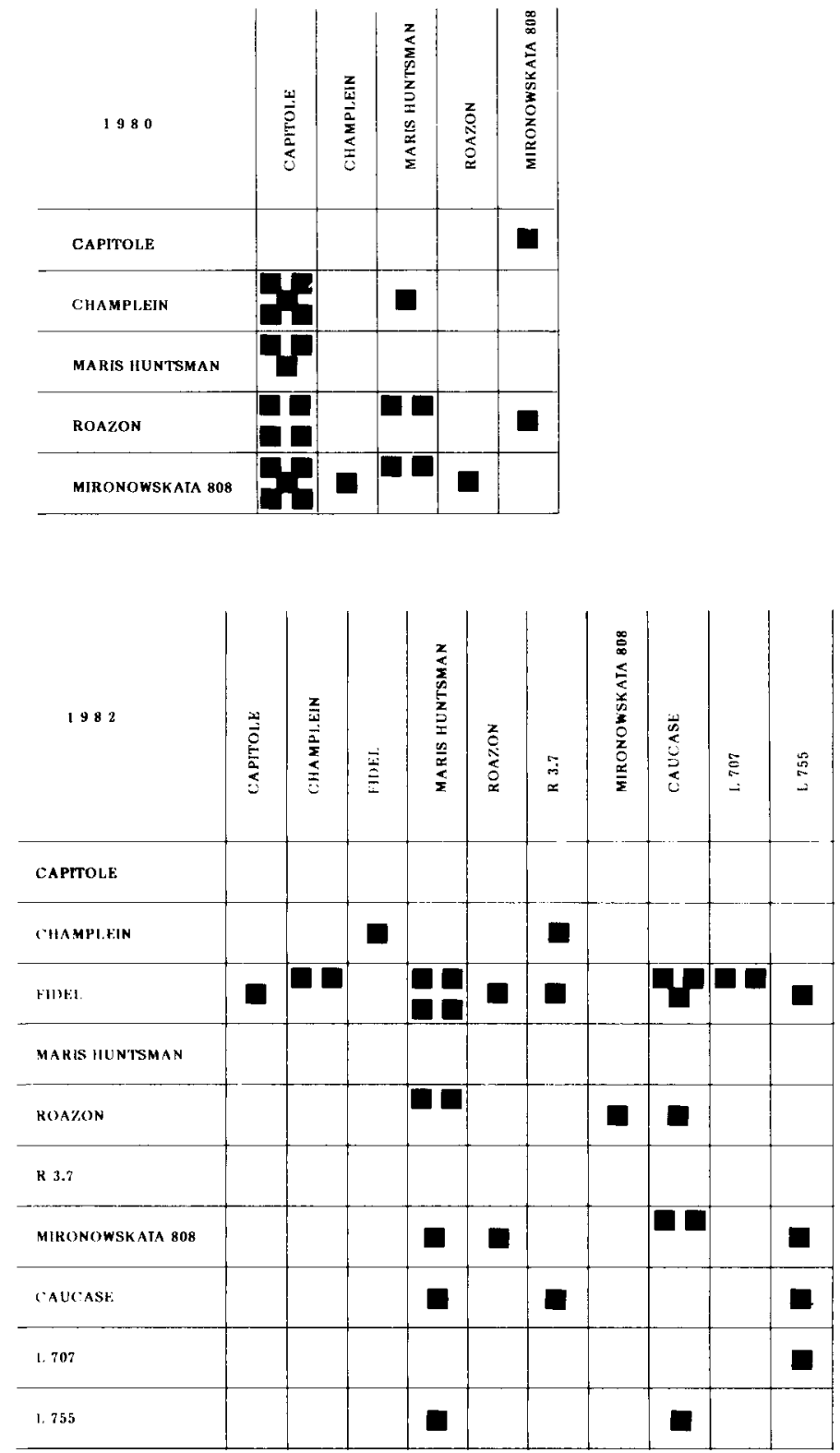

Duncan's range test on the factor "variety", for S. avenae. The number of symbols in each compartment of the tables indicates the number of observations for which a significant difference between 2 cultivars was found. The cultivar in the column is the more infested, the one in the row is the less infested.

vent et le plus fortement significatives. Les cultivars qui semblent particulièrement remarquables, c'est-àdire pour lesquels les tendances observées paraissent stables au cours des années, quelle que soit l'importance des infestations, sont "Maris Huntsman", presque toujours plus fortement infesté que les autres par au moins 2 , et souvent 3 espèces de pucerons, et "Roazon " qui, au contraire, est le plus souvent moins infesté que la plupart des autres variétés.

Par contre, "Capitole " est en 1978 et 1982 très faiblement infesté par les 3 espèces de pucerons, alors qu'il compte parmi les cultivars les plus infestés par les 3 espèces en 1980, et par $S$. avenae et $R$. padi en 1981. Cette instabilité des infestations se retrouve chez 

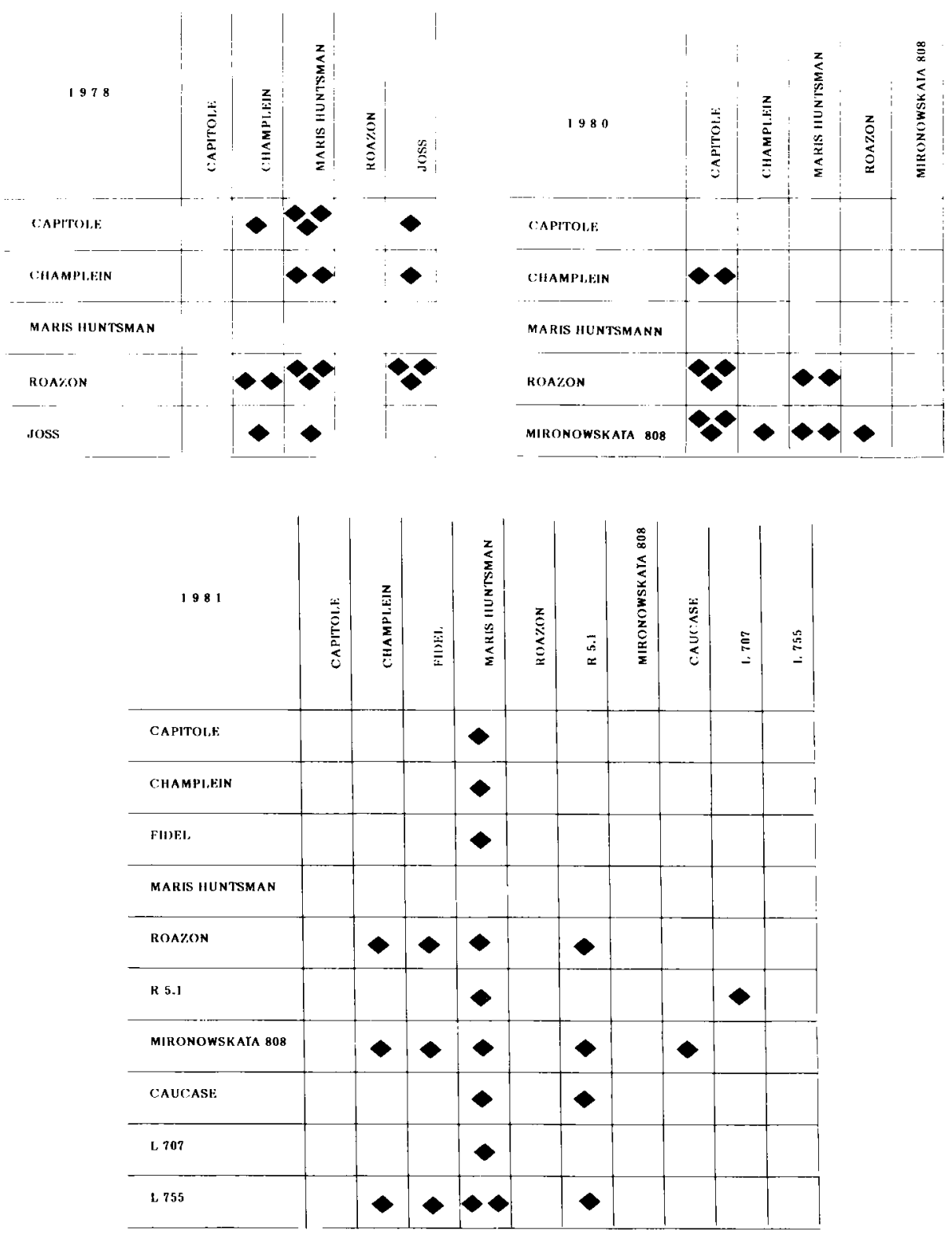

Figure 5

Même légende que pour la figure 4 pour $\mathrm{M}$. dirhodum.

« Mironowskaïa 808 » qui, décrit comme peu favorable aux pucerons, l'est effectivement dans certains cas (1980 pour les 3 espèces, 1981 pour $M$. dirhodum, 1982 pour $R$. padi) mais pas dans d'autres.

Enfin 2 cultivars qui n'ont été incorporés dans les expérimentations qu'en 1981 et 1982 retiennent l'attention : "Caucase ", assez infesté par $S$. avenae, et surtout «Fidel » qui apparaît très défavorable à ce même puceron. Cependant, le trop faible nombre d'années d'étude ne permet pas de conclure à leur sujet.

C. Etude détaillée de l'évolution des populations de $S$. avenae sur les cultivars "Maris Huntsman " et « Roazon "

Sur la figure 7 sont représentées les courbes d'évolution des populations de $S$. avenae, pendant 5 années consécutives, sur les 2 cultivars pour lesquels les différences d'infestation paraissent les plus stables dans le temps, au cours des années. Ces cultivars sont « Maris Huntsman » et " Roazon ».

Les populations sont, en début de saison, peu différentes l'une de l'autre (on a vu que les différences intervariétales ne sont jamais significatives à cette époque) ; elles augmentent jusqu'à ce que les cultures atteignent un stade phénologique compris, selon les années et les cultivars, entre 64 (mi-floraison) et 75 (mi-maturité laiteuse). Du fait des différences de précocité entre les 2 cultivars (« Maris Huntsman » est plutôt tardif alors que " Roazon » est précoce), les populations de pucerons continuent à augmenter sur "Maris Huntsman " alors qu'elles ont déjà entamé leur régression sur " Roazon » qui atteint environ une semaine plus tôt les stades considérés. Les différences de populations, au moment où celles-ci sont maximales, peuvent être importantes : 8 fois plus fortes sur "Maris Huntsman " que sur "Roazon » en 1978 et 1979 ; 2 à 3 fois plus fortes sur "Maris Huntsman » que sur « Roazon» les autres années. 

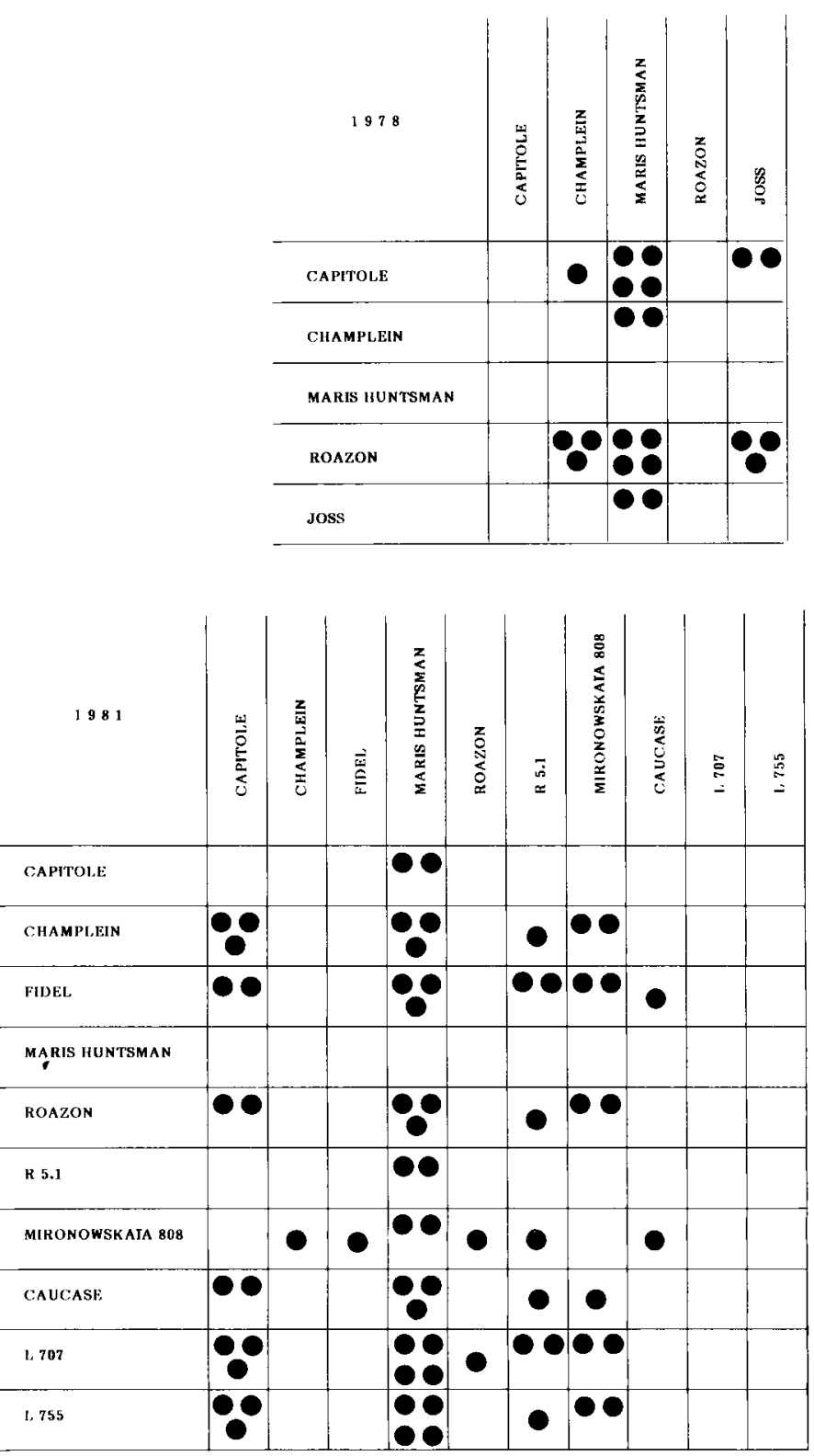

Figure 6

Même légende que pour la figure 4 pour $\mathrm{R}$. padi.

Il faut remarquer, en outre, que les courbes d'évolution des populations restent très proches l'une de l'autre, voire pratiquement confondues comme en 1980 , avant que la régression survenue sur « Roazon » les sépare : ce rythme de développement de $S$. avenae est très voisin sur les 2 cultivars tant que l'un d'eux n'atteint pas un état phénologique apparemment moins favorable au puceron.

Si les différences de précocité semblent être un élément d'explication des différences intervariétales observées dans le cas de "Roazon" et "Maris Huntsman », d'autres facteurs entrent probablement en jeu : en effet, pour ces 2 cultivars, la chute des populations ne s'amorce pas toujours exactement au même stade : 64 pour « Roazon " au lieu de 73 pour « Maris Huntsman » en 1978, 69 pour « Roazon » et 64 pour « Maris Huntsman » en 1982 ; à l'inverse, les populations stagnent, voire décroissent légèrement en 1980, entre les stades 69 et 75 dans le cas de « Maris
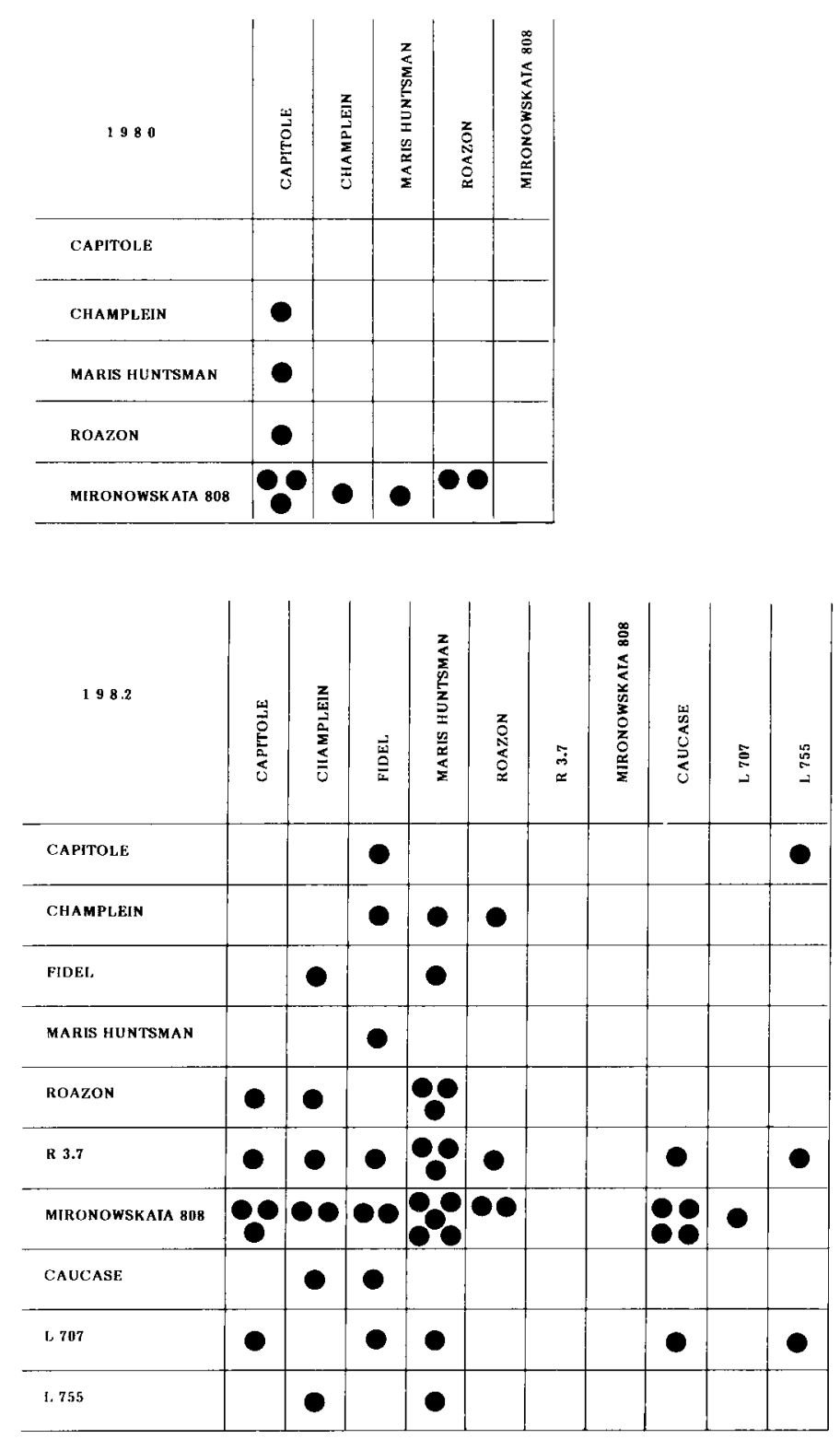

As for figure 4 for $\mathrm{R}$. padi.

Huntsman », alors qu'elles augmentent jusqu'au stade 75 pour « Roazon ». Il est probable que des raisons de nature pathologique accroissent certaines années l'effet strictement phénologique observé ou le remplacent lorsqu'il n'existe apparemment pas (1982). « Roazon » est assez sensible à diverses maladies foliaires, en particulier à l'oïdium, qui accélèrent le dessèchement des parties végétatives des plantes, alors que «Maris Huntsman » leur est plutôt résistant.

D. Importance de l'effet variétal dans la hiérarchie des facteurs de développement des populations de pucerons

L'indice Rautapäa (RAUTAPÄA, 1966), qui représente la somme journalière des populations de pucerons présentes sur un échantillon de taille fixe, donne 

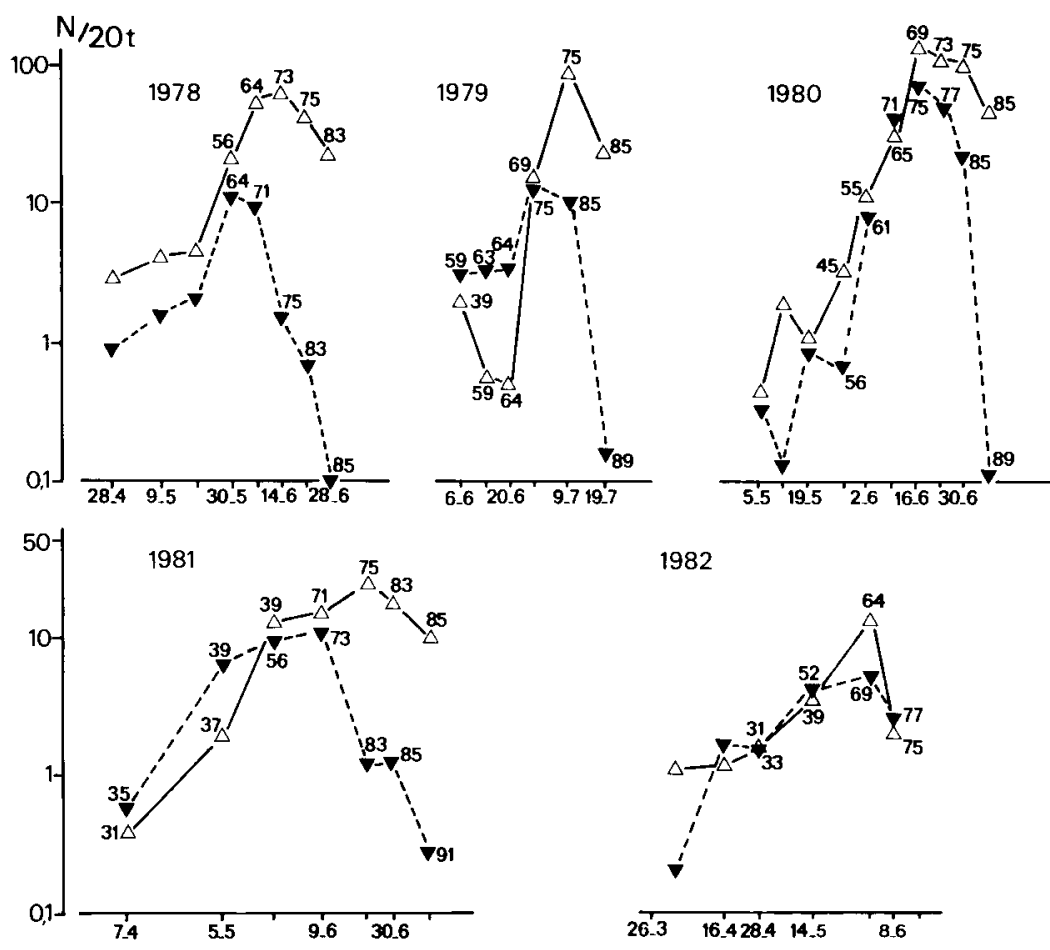

1982

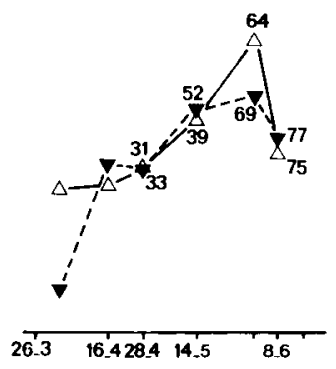

Figure 7

Evolution des populations de S. avenae sur "Maris Huntsman》 $(-\triangle-)$ et sur « Roazon » (-----) de 1978 à 1982. Les dates de prélèvement sont représentées en abscisses et les nombres de pucerons pour 20 talles en ordonnées.

une image de la population d'une espèce donnée supportée par un végétal au cours d'une période d'expérimentation ; il peut donc être considéré comme un indice d'infestation.

La figure 8 représente le plan factoriel d'une analyse des correspondances effectuée sur les indices Rautapäa calculés par échantillon de 20 talles, avec pour variables les 3 espèces de pucerons et pour individus 41 associations cultivar-année-date de semis ( 5 cultivars et une date de semis en 1978, 4 cultivars et 2 dates de semis en 1979, 5 cultivars et 2 dates de semis en 1980, 6 cultivars et 1 date de semis en 1981, 6 cultivars et 2 dates de semis en 1982).

L'axe horizontal représente 72 p. 100 de l'inertie totale, il oppose $R$. padi à $S$. avenae et $M$. dirhodum. L'axe vertical oppose $S$. avenae à $M$. dirhodum et représente 28 p. 100 de l'inertie totale.

Alors que les points correspondant à une même variété sont, dans la plupart des cas, très éloignés les uns des autres, les individus se regroupent assez bien en fonction de l'année et en fonction de la date de semis lorsque celle-ci a une importance écologique (infestation anté-hivernale des semis précoces et développement plus important des populations printanières sur ceux-ci), c'est-à-dire en 1980 et en 1982 (DEDRYVER \& TANGUY, 1984).

Un $1^{\text {er }}$ groupe de points comprend les 5 cultivars testés en 1978, qui sont fortement associés à l'espèce M. dirhodum. Trois autres groupes comprennent des points correspondant à des semis précoces (1980, 1981,1982 ), tous plus ou moins fortement associés à l'espèce $R$. padi. C'est particulièrement le cas pour l'année 1980 , en ce qui concerne 4 cultivars sur 5 .
Development of S. avenae populations on "Maris Huntsman 》 $(-\triangle-)$ and on "Roazon " (-- --$)$ from 1978 to 1982. Abscissa : dates of sampling. Qrdinate: number of aphids on 20 tillers.

A l'inverse, les points correspondant à des dates de semis tardives sont tous situés dans l'angle supérieur droit du plan factoriel et donc caractérisés par une association à $R$. padi très faible ou nulle. Il en découle l'éloignement des nuages de points correspondant, pour une même année, à des dates de semis différentes en 1980 et 1982, alors que ceux-ci sont imbriqués en 1979, année pour laquelle l'effet "date de semis" ne se manifeste pas, les semis dans toutes les parcelles étant assimilables à des semis tardifs (DEDRYVER \& TANGUY, 1984).

Par contre, pour chaque année et chaque date de semis, les points correspondant aux différents cultivars étudiés sont en général assez proches les uns des autres, ce qui indique que "l'effet variétal " sur les pucerons ne paraît pas être du même ordre de grandeur que celui dû à la date de semis et aux circonstances propres aux différentes années d'étude.

Ainsi, des effets variétaux mis en évidence aux paragraphes précédents, il apparaît seulement le « comportement " intéressant de "Mironowskaïa 808 " à la $1^{\text {re }}$ date de semis de 1980 , du fait de sa très faible infestation en $R$. padi par rapport aux 4 autres cultivars, abondamment colonisés par cette espèce. Dans une moindre mesure, on notera également la position particulière de "Fidel » en 1981, par rapport à celle des autres points de la même expérimentation.

Dans cette analyse, les effets variétaux ne paraissent s'exprimer qu'à l'intérieur d'ensembles plus différenciés déterminant des « situations moyennes », fonctions de la date de semis du blé (donc de l'époque de sa contamination) et, plus généralement, des circonstances écologiques de l'année d'expérimentation. 


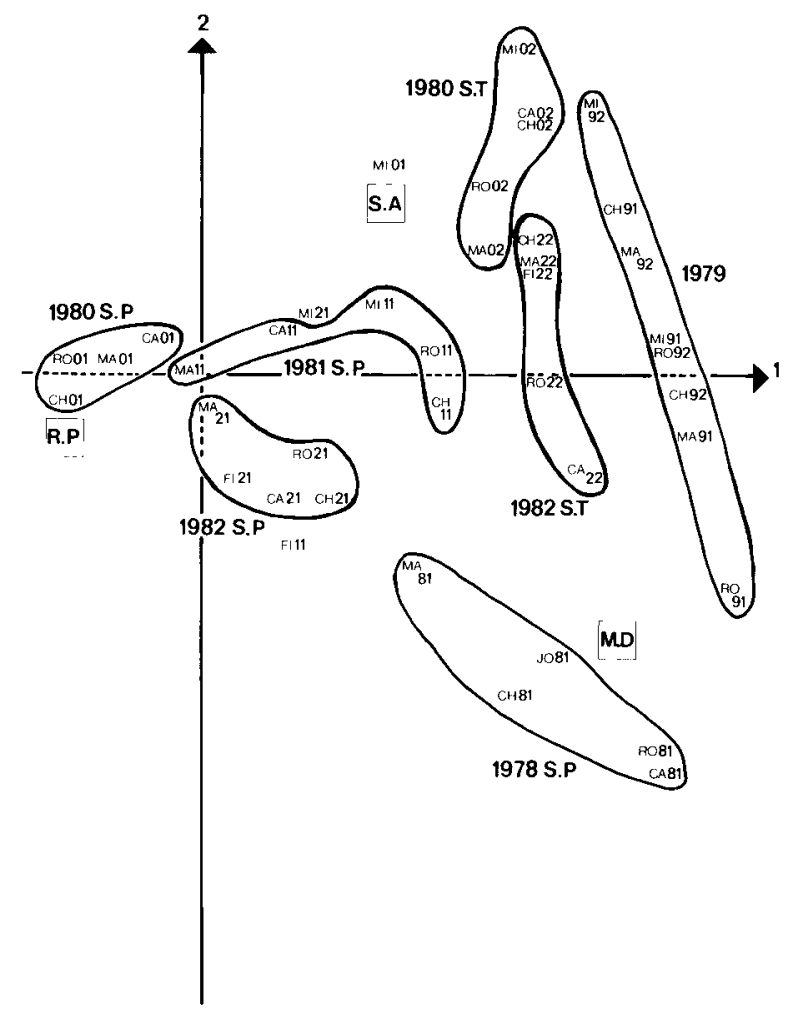

Figure 8

Plan factoriel d'une analyse des correspondances effectuées sur les indices Rautapää des populations de pucerons. Les individus sont les associations cultivar-année-date de semis. Les variables sont les espèces de pucerons: $S A$ : S. avenae, $M D$ : M. dirhodum, $R P$ : R. padi.

$S P$ : semis précoce, $S T:$ semis tardif.

$1: 1^{\text {er }}$ axe factoriel, $2: 2^{e}$ axe factoriel.

$\mathrm{CH}:$ : Champlein "

JO : « Joss"

$M A$ : «Maris Huntsman »

$C A:$ : Capitole»

RO : «Roazon

MI : « Mironowskaïa $808 »$

FI : " Fidel».

Le $1^{\text {er }}$ chiffre suivant les initiales de la variété indique l'année :

$$
8: 1978 ; 9: 1979 ; 0: 1980
$$

$1: 1981 ; 2: 1982$

Le $2^{e}$ indique la date de semis $1: 1^{r e}$

$$
2: 2^{e} \text {. }
$$

Plan of a factorial correspondance analysis on the Rautapäa index of aphid populations. Individuals are cultivar-year-sowing date associations. Variables are the species of aphids: $S A$ : S. avenae, $M D$ : M. dirhodum, $R P$ : R. padi.

$S P$ : early sowing, $S T$ : late sowing.

$I:$ first factorial axis, $2:$ second factorial axis.

$C H$ : « Champlein »

JO : « Joss»

$M A$ : «Maris Huntsman "

$C A:$ : Capitole»

$R O: \ll R o a z o n »$

FI : « Fidel».

The first number following the initials of the cultivar indicates the year :

$8: 1978 ; 9: 1979 ; 0: 1980$

$1: 1981 ; 2: 1982$.

The second indicates the sowing date $1: 1^{\text {st }}$

$$
2: 2^{\text {nd }}
$$

\section{DISCUSSION ET CONCLUSION}

Les résultats des types d'expérimentation en plein champ que nous avons utilisés tendent à mettre en évidence un effet global du cultivar sur la multiplication des populations de pucerons; les plus nets sont corroborés par d'autres travaux : c'est ainsi que LEE (comm. pers.), en Grande-Bretagne, considère « Maris Huntsman » comme fortement multiplicateur de pucerons au champ. En plein champ également, Chauveau (comm. pers.) trouve ce cultivar très contaminé par les pucerons, toutes espèces confondues, alors que pour DUFOUR (1982), il est essentiellement « sensible » à $M$. dirhodum. Ce même auteur a également mis en évidence la faible multiplication de $S$. avenae sur « Fidel ", l'attribuant à la forte aristation de l'épi. Nous avons déjà fait mention des travaux de HINZ \& DAEBELLER $(1974 ; 1976)$ signalant «Mironowskaïa 808 » comme peu favorable aux pucerons.

A l'exclusion de ces quelques "pôles de stabilité ", on observe une grande variation des résultats obtenus. Ainsi « Capitole » est considéré comme peu favorable aux pucerons, au cours d'une seule année d'expérimentation dans le Sud-Ouest de la France (CHAUVEAU, comm. pers.), alors que DUFOUR (1982) le trouve "faiblement sensible " à $R$. padi. Il en est de même pour « Roazon », considéré par cet auteur comme particulièrement infesté par $M$. dirhodum dans le Midi de la France alors que, globalement dans le Bassin de Rennes, c'est un des cultivars les moins infestés.

Cette variation des résultats obtenus peut s'expliquer par la diversité des facteurs en cause qui, d'une part, risquent de ne pas être les mêmes pour toutes les variétés étudiées et, d'autre part, peuvent intervenir différemment selon les conditions pédoclimatiques régionales.

- Il existe très certainement un effet phénologique dû à la différence de précocité des cultivars testés. En effet, le taux d'accroissement intrinsèque journalier des pucerons augmente au cours de la croissance du blé à partir du stade plantule vernalisée (DI PIETRO \& AKLI, travaux non publiés), passe par un maximum au stade maturité laiteuse puis décroît fortement à la maturité pâteuse. Dans le contexte climatique de l'Ouest de la France, en particulier dans le cas de semis précoces, des différences phénologiques importantes existent entre cultivars aux époques favorables à la multiplication des pucerons, ce qui est un élément d'explication des différences enregistrées dans les niveaux de population à des périodes données.

- L'influence de l'état sanitaire de la plante semble importante : il est significatif que ce soient les années où l'état pathologique des blés a été, dans l'ensemble, le plus mauvais $(1978,1981)$ que les différences intervariétales ont été les plus fortes, peut-être en partie parce que les cultivars assez résistants aux maladies foliaires comme "Maris Huntsman ", se sont trouvés dans une situation sanitaire bien meilleure que d'autres comme "Roazon». Ceci n'est qu'une hypothèse ; en effet, nous n'avons pu mesurer précisément l'état sanitaire et physiologique des plantes qui ont fait l'objet de nos expérimentations et il n'y a pas, à notre connaissance, de données bibliographiques se rapportant à ce sujet précis.

- Il paraît y avoir, au moins dans certains cas, une "résistance vraie " (antibiosis) de la plante, dont l'effet, selon les cas, peut s'ajouter aux effets précédents ou s'en retrancher : des expérimentations effectuées en conditions contrôlées (DI PIETRO \& AKLI, 
travaux non publiés) ont montré que le taux d'accroissement intrinsèque journalier (r) de $S$. avenae différait sensiblement selon les cultivars quel que soit le stade du blé, les différences les plus fortes se situant entre le tallage et l'épiaison.

Les conséquences agronomiques ainsi mises en évidence peuvent être envisagées à 2 échelles différentes :

- Au niveau de l'état phytosanitaire de la culture : l'intérêt de cultivars peu ou moins infestés que d'autres par les pucerons dépend de l'origine des résistances observées. Alors qu'une résistance vraie (même partielle) semble intéressante, il n'est pas envisageable de conseiller des variétés moins infestées par les pucerons si elles sont sensibles aux maladies ou mal adaptées ( Mironowskaïa 808 ») aux conditions agronomiques locales. Enfin, bien que les recherches concernant la nuisibilité directe des pucerons des céréales aient fait récemment de gros progrès (LEE et al., 1981) rien, jusqu'à présent, ne permet d'affirmer que des cultivars différents réagissent de manière semblable à une même quantité de pucerons.

- Au niveau régional : le problème des pucerons des céréales doit être envisagé à l'échelle de l'assolement. Au sein d'une petite région agricole, le nombre de variétés de blé d'hiver cultivées est toujours très faible et, dans la plupart des cas, une ou 2 d'entre elles, très dominantes de par les surfaces emblavées. Le fait qu'un de ces cultivars très répandus soit peu ou au contraire fortement infesté par les pucerons ne sera pas sans conséquences sur la biomasse de pucerons produite à l'échelle de la région considérée et donc sur les fluctuations de populations de ces ravageurs ainsi que sur les niveaux d'infestation d'autres graminées qui leur sont également sensibles.

Ce travail de terrain est complété par l'analyse en conditions contrôlées des différentes composantes du taux d'accroissement potentiel des pucerons sur ces mêmes cultivars, de manière à isoler les phénomènes de "résistance » ou de " sensibilité » aux pucerons d'autres facteurs pouvant agir sur leur multiplication, comme la phénologie ou la sensibilité aux maladies des différents cultivars. Ces expérimentations de laboratoire feront l'objet d'un prochain article.

Recu le 21 mars 1985. Accepté le 23 août 1985.

\section{REMERCIEMENTS}

Les auteurs tiennent à remercier vivement $G$. Doussinault de la Station d'Amélioration des Plantes I.N.R.A. du Rheu, pour l'aide qu'il leur a accordée.

\section{RÉFÉRENCES BIBLIOGRAPHIQUES}

Campbell B., Mc Lean D., Kinsey M., Jones K., Dreyer D., 1982 Probing behavior of the greenbug (Schizaphis graminum, biotype C) on resistant and susceptible varieties of sorghum. Entomol. exp. appl., 31, 140-146.

Dedryver C. A., Tanguy S., 1984. Biologie des pucerons des céréales dans l'Ouest de la France. V - Influence de la date de semis du blé d'hiver sur les modalités d'infestation des parcelles par Rhopalosiphum padi L., Sitobion avenae F., et Metopolophium dirhodum Wlk. et sur le développement de leurs populations au printemps. Agronomie, 4 (8), 711-719.

Di Pietro J.-P., Dedryver C. A., 1984. Quelques facteurs conditionnant l'expression du potentiel biotique des pucerons des céréales sur différents cultivars de blé d'hiver. $O I L B$, VII (4), 11-14.

Dufour B., 1982. Les pucerons des céréales dans la région de Montpellier. Dynamique des populations et facteurs de régulation. Thèse $3^{\mathrm{e}}$ cycle, Université des Sciences et Techniques du Languedoc, 178 p.

Harvey T., Martin T., Livers W., 1980. Resistance to biotype C greenbug in synthetic hexaploid wheats derived from Triticum tauschii. Entomol. Soc. America, 73 (3), 387-389.

Hinz B., Daebeller F., 1974. Untersuchungen zur Anfälligkeit verschiedener Getreidearten und Sorten gegenüber Getreideblattläusen. Arch. Phytopathol. Pflanzenschutz, 10 (5), 341-346.

Hinz B., Daebeller F., 1976. Zur Beeinflussung der Ertragsbildung bei Wintereizensorten durch die grosse Getreideblattlaus Macrosiphum (Sitobion) avenae F. Arch. Phytopathol. Pflanzenschutz, 12 (2) 111-116.

Joppa L., Timian R., Williams N., 1980. Inheritance of resistance to greenbug toxicity in an amphiploid of Triticum turgidum T. tauschii. Crop Sci., 20 (3), 343-344.
Latteur G., 1976. Les pucerons des céréales : biologie, nuisance, ennemis. Mémoire $N^{\circ} 3$ C.R.A. Gembloux, 74 p.

Lee G., Stevens D. J., Stokes S., Wratten S. D., 1981. Duration of cereal aphid populations and the effects on wheat yield and breadmaking quality. Ann. appl. Biol., 98, 169-178.

Lowe H. J. B., 1978. Detection of resistance to aphids in cereals. Ann. appl. Biol., 88, 401-406.

Lowe H. J. B., 1980. Resistance to aphids in immature wheat and barley. Ann. appl. Biol., 95, 129-135.

Lowe H. J. B., 1981a. Resistance and susceptibility to colour forms of the aphid Sitobion avenae in spring and winter wheats (Triticum aestivum). Ann. appl. Bıol., 99, 87-98.

Lowe H. J. B., 1981b. Resistance to Sitobion avenae in wheat. IOBC/WPRS Bull., 1981/IV/1, 47-50.

Lowe H. J. B., 1982. Resistance to aphids. Annu. Rep. Plant Breeding Instit. for 1981, 99-101.

Morgan J., Wilde G., Johnson D., 1980. Greenbug resistance in commercial sorghum hybrids in the seedling stage. J. econ. Entomol., 73 (4).

Rautapää J., 1966. The effect of the English grain aphid $M$. avenae on the yield and quality of wheat. Ann. Agric. Fenn., 5, 334-341.

Sotherton N. W., Van Emden H. F., 1982. Laboratory assessments of resistance to the aphid Sitobion avenae and Metopolophium dirhodum in three Triticum species and two modern wheat cultivars. Ann. appl. Biol., 101, 99-107.

Zadoks J. C., Chang T. T., Konzak C. F., 1977. Un code décimal pour les stades de croissance des céréales. Phytiatr.-Phytopharm., 26, $129-140$. 